\title{
EVALUACIÓN DE LAS ALTERACIONES EN EL DESEMPEÑO COGNITIVO DE NIÑOS MEXICANOS EXPUESTOS A PLAGUICIDAS ORGANOFOSFORADOS
}

\author{
Leticia YÁÑEZ-ESTRADA ${ }^{1 *}$, Ma. del Rocío RAMÍREZ-JIMÉNEZ ${ }^{1,2}$, Yaneth RODRÍGUEZ-AGUDELO $^{3}$, \\ Jaqueline CALDERÓN-HERNÁNDEZ ${ }^{4}$ y Edith RAMOS-RUIZ ${ }^{1}$
}

${ }^{1}$ Laboratorio de Género, Salud y Ambiente, Universidad Autónoma de San Luis Potosí

${ }^{2}$ Centro Interdisciplinario de Investigación para el Desarrollo Integral Regional-Unidad Michoacán, Instituto Politécnico Nacional

${ }^{3}$ Departamento de Neuropsicología, Instituto Nacional de Neurología y Neurocirugía "Manuel Velasco Suárez"

${ }^{4}$ Centro de Investigación Aplicada en Ambiente y Salud, CIACYT-Medicina, Universidad Autónoma de San Luis Potosí

*Autora para correspondencia: lyanez@uaslp.mx

(Recibido enero 2017; aceptado mayo 2018)

Palabras clave: plaguicidas organofosforados, coeficiente intelectual, infantes

\section{RESUMEN}

Los organofosforados $(\mathrm{OP})$ son los plaguicidas más comúnmente empleados en México. Estos agroquímicos son neurotóxicos y se han asociado con déficits neuroconductuales; sin embargo, pocos estudios realizados en México han evaluado la correlación entre las concentraciones urinarias de los metabolitos dialquilfosfatos y el desempeño cognitivo en niños. El objetivo de este estudio fue evaluar las alteraciones cognitivas en niños expuestos a OP de una localidad agrícola de México. Se recolectaron muestras de orina y sangre de 105 niños (de 5 a 14 años de edad) durante un periodo de alta aplicación de plaguicidas y se midieron los niveles urinarios de los metabolitos dialquilfosfatos (DAP) para evaluar la exposición a OP, así como las concentraciones de plomo en sangre, como factor confusor. Las madres de los niños fueron entrevistadas sobre aspectos de estilo de vida y otras variables de interés. El desempeño cognitivo de los niños fue evaluado por neuropsicólogos empleando la Escala Wechsler de Inteligencia para Niños-IV. La exposición a OP estuvo asociada con bajo desempeño cognitivo. Los niños del quintil con la mayor concentración de DAP presentaron un déficit de 12 puntos en el coeficiente intelectual, en comparación con los niños del quintil con la menor concentración de estos metabolitos ( $>312.1 \mathrm{nmol} / \mathrm{L}$ vs. $\leq 150.9 \mathrm{nmol} / \mathrm{L})$. De las subescalas evaluadas, el Índice de Memoria de Trabajo presentó las puntuaciones más bajas (media: 88.2). Los resultados sugieren que los niños de localidades agrícolas expuestos a $\mathrm{OP}$ tienen mayor riesgo de presentar alteraciones cognitivas.

Key words: organophosphorus pesticides, IQ, infants

\begin{abstract}
Organophosphorus (OPs) compounds are the most commonly used pesticides in México. These agrochemicals are neurotoxic and have been associated with neurobehavioral deficits; however, few studies carried out in Mexico have assessed the
\end{abstract}


correlation between urinary concentrations of dialkylphosphate metabolites and cognitive performance in children. The objective of this study was to evaluate cognitive alterations in children exposed to OPs from an agricultural locality in México. Urine and blood samples of 105 children (5-14 yrs of age) were collected during a period of high pesticide application; urinary levels of dialkylphosphate metabolites (DAPs) were measured to evaluate exposure to OPs and lead concentrations in blood were determined as a confusing factor. The mothers of the children were interviewed on aspects of lifestyle and other variables of interest. The cognitive performance of the children was assessed by neuropsychologists, using the Wechsler scale of intelligence for children-IV. OPs exposure was associated with low cognitive performance. The children of the quintile with the highest concentration of DAPs, presented an IQ deficit of 12 points, compared with children of the quintile with the lowest concentration of these ( $>312.1 \mathrm{nmol} / 1 \mathrm{vs} . \leq 150.9 \mathrm{nmol} / \mathrm{L}$ ). Of the subscales evaluated, the working memory index presented the lowest scores (mean: 88.2). The results suggest that children of agricultural localities exposed to OPs have a higher risk of presenting cognitive alterations.

\section{INTRODUCCIÓN}

En la actualidad, los plaguicidas organofosforados (OP) se encuentran entre los compuestos más utilizados en México, principalmente en actividades agrícolas, no obstante su probado efecto neurotóxico (Quintanilla-Vega et al. 2010, Sánchez-Guerra et al. 2011, Androutsopoulos et al. 2012). El metabolismo oxidativo de estos compuestos se lleva a cabo principalmente en el hígado a través del citocromo P450, formando el oxón correspondiente, que es la estructura química que ejerce la acción tóxica. La principal ruta de desintoxicación de estos compuestos consiste en la hidrólisis de este oxón por la paraoxonasa 1 (Costa et al. 2003, 2005), la cual produce alcohol además de los metabolitos dialquilfosfatos (DAP). Éstos se agrupan de acuerdo con su estructura química y el compuesto organofosforado padre, formando metilados constituidos por dimetilfosfato (DMP), dimetiltiofosfato (DMTP) y dimetilditiofosfato (DMDTP), y etilados constituidos por dietilfosfato (DEP), dietiltiofosfato (DETP) y dietiditiofosfato (DEDTP). Por ello la exposición a los plaguicidas OP se estima a través de la medición urinaria de estos metabolitos (Lambert et al. 2005, Valcke et al. 2006, Arcury et al. 2007). Las vías de exposición para aquellos que viven en localidades agrícolas son la inhalación de aire contaminado tanto en ambientes interiores como exteriores, la ingesta de alimentos o agua contaminados, y el contacto dérmico con materiales y superficies contaminadas con estos compuestos, entre otras (Vida y Moretto 2007). Diferentes estudios epidemiológicos han reportado que las concentraciones urinarias de metabolitos DAP son superiores en niños que viven en zonas agrícolas, en comparación con aquellos que residen en áreas urbanas (Koch et al. 2002, Barr et al. 2004, Lambert et al. 2005, Valcke et al. 2006, Arcury et al. 2007, Oulhote y Bouchard 2013).

Los reportes en la literatura sobre los efectos neurotóxicos de estos agroquímicos en poblaciones humanas se centran principalmente en escenarios ocupacionales y exposiciones agudas, siendo escasos los estudios en población abierta expuesta de forma crónica (Kamel et al. 2007).

Los niños son particularmente vulnerables a los efectos tóxicos por la exposición a contaminantes debido a diversos factores fisiológicos, entre los cuales están sus mecanismos inmaduros de destoxificación (Weiss et al. 2004, Sly y Flack 2008). Algunos estudios realizados en niños han informado asociaciones entre exposición a plaguicidas OP y efectos neurológicos (Guillette et al. 1998, Ruckart et al. 2004, Rohlman et al. 2005, Young et al. 2005, Grandjean et al. 2006, Rauh et al. 2006, Engel et al. 2007, 2011, Eskenazi et al. 2007), en diversos elementos como abstracción y síntesis verbal, atención, memoria a corto y largo plazo, velocidad de procesamiento, coordinación motriz, y rasgos de hiperactividad e impulsividad.

Si bien es cierto que en la literatura ya se ha reportado el efecto neurotóxico de los plaguicidas OP en niños expuestos a éstos, en México son escasos los estudios al respecto (Gamlin et al. 2006, Quintanilla et al. 2010, Sánchez-Guerra et al. 2011, Rivero Pérez 2012), no obstante que la población infantil es de aproximadamente 32.5 millones (29\% de la población total), de los cuales el $26.6 \%$ vive en localidades rurales (INEGI 2010). Además, en algunos de estos estudios no se estimó la exposición a 
agroquímicos por medio de biomarcadores para evaluar su magnitud, ni se consideraron factores confusores como la exposición a otros compuestos neurotóxicos (como plomo) o la escolaridad de la madre, de ahí que el objetivo del presente estudio fue evaluar las alteraciones en el desempeño cognitivo de niños mexicanos expuestos crónicamente a plaguicidas organofosforados, considerando la función escolar de la madre, los niveles de plomo en sangre, y la cuantificación de DAP en orina para estimar la magnitud de la exposición infantil a los plaguicidas OP.

\section{MATERIALES Y MÉTODOS}

\section{Área y población de estudio}

La localidad de El Refugio está localizada en el municipio de Ciudad Fernández, en una de las zonas agrícolas más importantes del estado de San Luis Potosí, México (Fig. 1), por lo que en la región se aplica una gran diversidad de plaguicidas (OP, piretroides, carbamatos y organoclorados, entre otros). De acuerdo con lo indicado por agricultores del área de estudio, entre los plaguicidas OP más usados se encuentran los siguientes: clorpirifos etílico, diazinón, malatión, metamidofos, paratión metílico, dimetoato, acefato, etión y monocrotofos, entre otros.

Para la selección de los niños participantes se visitó una escuela primaria ubicada en el centro de la localidad y por ende alejada de las parcelas (escuela A) y otra que se encuentra en la periferia, más próxima a los campos de cultivo (escuela $\mathrm{B}$ ). En ambos centros educativos se organizaron charlas con los padres de familia para explicarles los objetivos del estudio, los beneficios que recibirían como un diagnóstico sencillo de salud de sus hijos, que el estudio era gratuito, que su participación era voluntaria y que podrían retirarse del mismo cuando así lo consideraran pertinente sin dar explicación alguna, recibiendo todos los beneficios hasta el día de su colaboración. La aceptación por parte de los padres de familia para que sus hijos fuesen incluidos en el estudio se dio mediante una firma del consentimiento informado. De esta manera, la población de estudio quedó conformada por 48 niños de la escuela A y 57 de la escuela B, entre 5 y 14 años de edad, clínicamente sanos (reportados por la madre y de acuerdo con los resultados de los análisis bioquímico-clínicos (datos no mostrados() y con un tiempo de residencia en la localidad de al menos 5 años. El trabajo fue aprobado por el Comité de Ética en Investigación de

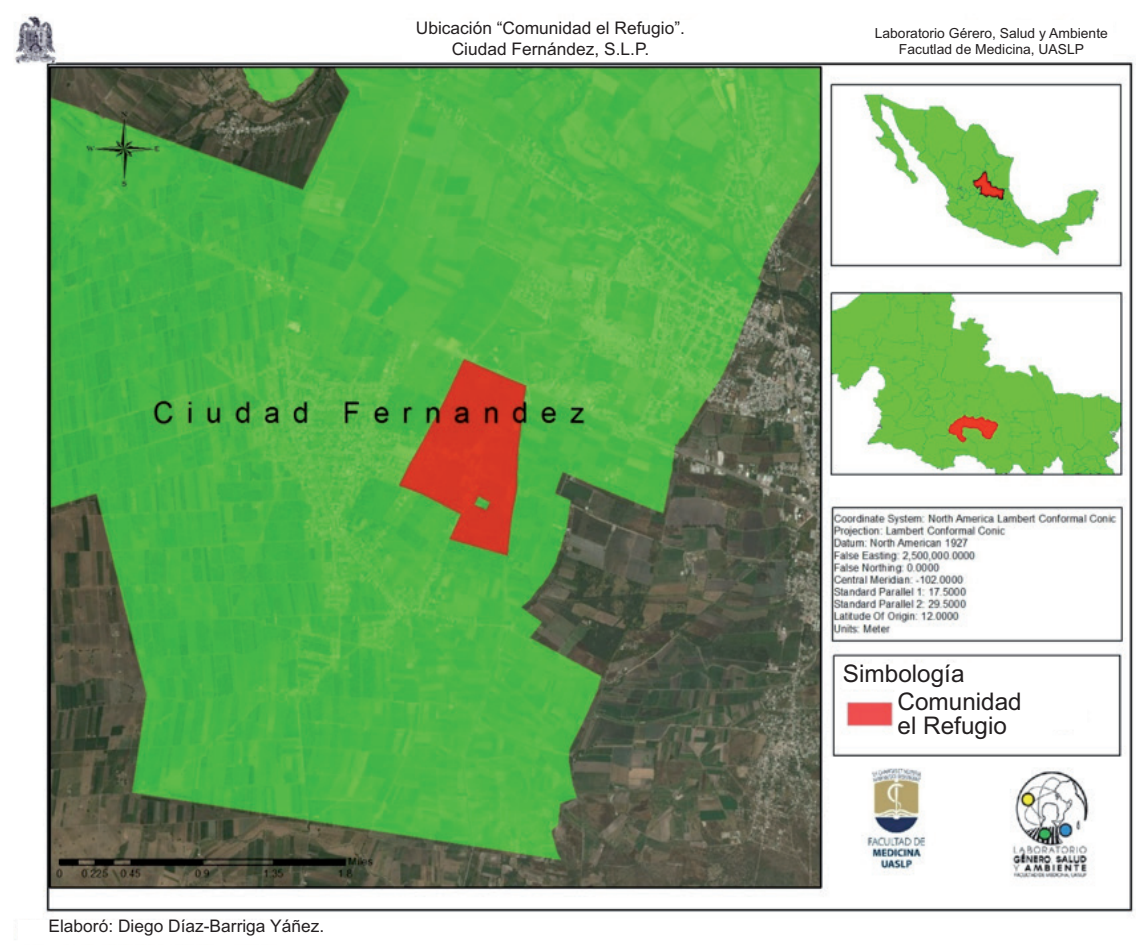

Fig. 1. Localización del sitio de estudio: El Refugio, Ciudad Fernández, San Luis Potosí, México 
la Facultad de Medicina de la Universidad Autónoma de San Luis Potosí (UASLP). El diseño del estudio fue transversal y se realizó durante un periodo de alta aplicación de plaguicidas (mayo de 2010).

\section{Recolección de las muestras biológicas}

La recolección de las muestras de sangre fue realizada por personal especializado, empleando para ello material nuevo y estéril. Se recolectaron en ayunas (entre las 7:45 y 9:15 h) $4 \mathrm{~mL}$ de sangre por punción venosa en un tubo BD Vacutainer ${ }^{\circledR}$ con EDTA para la determinación de plomo (como factor confusor de daño neurológico) y para realizar una biometría hemática.

Para cuantificar los niveles de los metabolitos urinarios DAP, de cada niño se recolectó la primera orina de la mañana durante cinco días consecutivos (dado que su vida media de eliminación es de 12 a $48 \mathrm{~h}$ (Costa 2013), en recipientes de plástico nuevos y estériles. Cada día, inmediatamente después de su obtención, las muestras fueron trasladadas en condiciones controladas de temperatura y luz al Laboratorio de Género, Salud y Ambiente de la Facultad de Medicina de la UASLP, donde se mantuvieron en refrigeración a $5{ }^{\circ} \mathrm{C}$. Al término del periodo de recolección, se conformó una muestra compuesta que se almacenó a $-70^{\circ} \mathrm{C}$ hasta su análisis.

\section{Determinación de plomo en sangre}

La determinación de plomo en sangre se realizó por espectrofotometría de absorción atómica (Perkin-Elmer modelo 3110, equipado con un horno de grafito) de acuerdo con el método descrito por Subramanian (1987). Como control de calidad se empleó un estándar de sangre (CDC 04PB39) del Centro de Control y Prevención de Enfermedades de Estados Unidos (CDC, por sus siglas en inglés).

\section{Análisis de los metabolitos DAP en orina}

La exposición a plaguicidas OF fue evaluada mediante la medición de los seis principales metabolitos urinarios DAP: DMP, DMTP, DMDTP, DEP, DETP y DEDTP, de acuerdo con la metodología descrita por Ramírez-Jiménez (2015). Para ello se empleó un cromatógrafo de gases-masas Agilent (modelo 6850 NS y $5973 \mathrm{~N}$ ), operado en el modo de monitoreo de ión selectivo y se eligió el dietiltiofosfato deuterado como estándar interno. Dada la naturaleza polar de estos compuestos, éstos fueron primero sometidos a una reacción de derivatización previo al proceso de extracción. Las concentraciones urinarias de los seis metabolitos diaquilfosfato se expresan en $\mathrm{nmol} / \mathrm{L} \mathrm{y}$ en $\mathrm{nmol} / \mathrm{g}$ creatinina, y se agrupan como metabolitos dimetil DMP (DMP, DMTP y DMDTP), metabolitos dietil DEP (DEP, DETP y DEDTP) y DAP (suma de DMP y DEP).

Los límites de detección (LD) del método analítico fueron: 1.36, 1.49, 0.27, 1.07, 0.98 y $0.52 \mu \mathrm{g} / \mathrm{L}$ para DMP, DMTP, DMDTP, DEP, DETP y DEDTP, respectivamente. Los porcentajes de recobro de los seis metabolitos fluctuaron entre el 92 y el $111 \%$. Las curvas de calibración de los seis metabolitos fueron lineales $(r \geq 0.993)$. Para fines estadísticos, los niveles $<$ LD fueron sustituidos por $\mathrm{LD} / \sqrt{ } 2$ (Barr et al. 2004).

Todas las determinaciones se realizaron en el Laboratorio de Género, Salud y Ambiente de la Facultad de Medicina de la UASLP, siguiendo los protocolos de control de calidad establecidos por el mismo laboratorio (elaboración de curvas de control de calidad, repetibilidad, reproducibilidad, inclusión de un estándar de concentración conocida por cada lote de 10 muestras procesadas).

La biometría hemática se realizó con un método automatizado empleando el autoanalizador hematológico KX21N (valores de referencia: 12 a $18 \mathrm{~g} / \mathrm{dL}$ ) y la concentración de creatinina en orina se determinó de acuerdo con el método colorimétrico basado en la reacción Jaffe (creatinina, procedimiento núm. 555, Sigma Diagnostics, St. Louis, Missouri).

\section{Medidas antropométricas y datos recabados a través de la entrevista-cuestionario}

Con el apoyo de una enfermera, el día de la recolección de muestras sanguíneas se determinaron los datos antropométricos (peso y talla) de los niños participantes en el estudio. Para el registro del peso se empleó una báscula electrónica, mientras que para la talla un estadímetro (ambos sin zapatos). Mediante el uso del software AnthroPlus v. 1.0.4., se calcularon el índice de masa corporal $\left(\mathrm{kg} / \mathrm{m}^{2}\right)$ y los puntajes $z$ de estatura para la edad e índice de masa corporal para la edad (de Onis et al. 2007). La prevalencia de bajo peso, peso normal, sobrepeso y obesidad fue determinada usando los valores de corte del IMC por edad y sexo del Grupo de Trabajo sobre Obesidad (IOTF por sus siglas en inglés) de la Organización Mundial de la Salud (Cole et al. 2000, 2007).

Para realizar la entrevista a las madres y recabar la información relacionada con ella y su hijo (características del embarazo y parto del hijo en estudio, así como si recibió seno materno, enfermedades más frecuentes, vacunas aplicadas al niño y años escolares no aprobados, entre otros); usos y costumbres de la familia (frecuencia de ingesta y tipo de alimento, sitio de adquisición de alimentos, combustible empleado 
en la cocción de los alimentos, actividades extraescolares del hijo y lugares comunes de juego, entre otros), características sociodemográficas generales (tipo de casa-habitación, material de construcción, número de cuartos y focos, acceso a servicios públicos, cercanía de la vivienda a las parcelas e ingreso económico mensual familiar entre otros), experiencia de exposición a insecticidas tanto de uso doméstico como de uso por el sector salud (para el control de enfermedades transmitidas por vectores, como el dengue y el paludismo), experiencia de exposición a agroquímicos, así como ocupación, consumo de alcohol y tabaco de los padres de familia, se diseñó un cuestionario y la investigadora principal capacitó a estudiantes de posgrado que participaron en el estudio para su aplicación.

\section{Evaluación neuropsicológica}

Durante la semana que se recolectaron las muestras de orina, un grupo de neuropsicólogos del Instituto Nacional de Neurología y Neurocirugía "Manuel Velasco Suárez" (INNNMVS), aplicaron a los niños participantes en el estudio, la escala de Wechsler de inteligencia para niños-IV (WISC-IV, por sus siglas en inglés) adaptada para población mexicana, conformada por un conjunto de subpruebas que evalúa diferentes habilidades cognitivas englobadas en los siguientes índices: índice de comprensión verbal (ICV), integrado por subpruebas de vocabulario y semejanzas; índice de razonamiento perceptual (IRP), integrado por subpruebas de diseño con cubos y matrices de razonamiento; índice de memoria de trabajo (IMT), integrado por subpruebas de retención de dígitos y de secuenciación de letras y números; e índice de velocidad de procesamiento (IVP), integrado por subpruebas de claves y búsqueda de símbolos. El coeficiente intelectual (CI) se estimó mediante la sumatoria del puntaje obtenido en cada una de las subescalas ajustado por la edad del niño (Wechsler 2003). Las pruebas fueron aplicadas en sesiones individuales a cada uno de los niños, con una duración aproximada de $45 \mathrm{~min}$.

Debido a que la función intelectual de la madre es un factor determinante en el desempeño del niño (Mink et al. 2004), ésta fue evaluada mediante la prueba de matrices progresivas de Raven (Raven 1993), la cual mide la capacidad intelectual a través el uso del razonamiento analógico por medio de una serie de figuras geométricas abstractas incompletas.

\section{Análisis estadístico}

Mediante la prueba de Kolmogorov-Smirnov $(\mathrm{p}<0.05)$ se evaluó la normalidad de las variables continuas, tales como el IMC para la edad, el nivel de plomo en sangre, las concentraciones urinarias de los metabolitos DAP (DMP, DEP y DAP), el nivel de creatinina urinaria, el nivel de hemoglobina, puntuaciones de las subescalas cognitivas y el CI, entre otras, y se calcularon los parámetros descriptivos de dichas variables (media, desviación estándar, percentiles, mínimo y máximo). Aquellas variables que no se ajustaron a una distribución normal se transformaron a $\log 10$.

Los ICV, IRP, IMT y IVP, así como el CI, fueron considerados como variables dependientes; mientras que las concentraciones de los metabolitos DMP, DEP y DAP como variables independientes. Como covariables se evaluaron la edad, el sexo, el valor del puntaje $z$ del IMC, el nivel de hemoglobina y la concentración de plomo en sangre de los niños, así como la edad, la escolaridad y la función intelectual de las madres. Las puntuaciones de los índices cognitivos y el CI se compararon con las concentraciones de los metabolitos DMP, DEP y DAP (estratificadas en quintiles) mediante la prueba ANOVA. Otras variables categóricas (sexo, ocupación del padre, exposición residencial a plaguicidas y proximidad de los campos de cultivo al hogar, entre otras) fueron evaluadas mediante las pruebas $t$ de Student o $U$ de Mann-Whitney. Con relación a la variable "proximidad de los campos de cultivo al hogar", durante la entrevista a las madres se registró el domicilio de la casa-habitación, ubicándose geográficamente mediante el software ARCGIS, con el cual también se estimó la distancia entre la vivienda y el campo de cultivo. Si ésta era igual o menor a $15 \mathrm{~m}$, se consideró que el hogar estaba próximo a la parcela.

Finalmente, se emplearon modelos de regresión lineal múltiple (MRLM) independientes para evaluar la asociación entre los índices cognitivos (CV, RP, MT, VP y CI total) y los tres grupos de metabolitos (DMP, DEP y DAP). Los valores de los metabolitos se estratificaron por quintiles y se introdujeron como variables ordinales en los MRLM: el primer quintil representa menor exposición y en el último mayor exposición. Los MRLM fueron ajustados por la función intelectual de la madre, el ingreso económico mensual familiar, la concentración de plomo en sangre, el puntaje $z$ de IMC para la edad y el nivel de hemoglobina. Las variables con $p$ igual o menor a 0.20 en el análisis bivariado o aquellas relevantes desde el punto de vista biológico, independientemente de la significancia estadística (plomo en sangre y hemoglobina), fueron incluidas en los MRLM. El análisis estadístico se realizó con el paquete 
estadístico SPSS (Statistical Package for Social Sciences) v. 18 para Windows (SPSS, Chicago, IL). El nivel de significancia que se estableció fue menor a 0.05 .

\section{RESULTADOS}

Las características generales de la población de estudio se muestran en el cuadro I. La edad promedio de los niños fue de $9.7 \pm 1.9$ años con un intervalo de 5 a 14 años, de los cuales el $49.5 \%$ eran niñas, el $89.5 \%$ nació en la zona de estudio y la totalidad tenía una residencia en la comunidad agrícola igual o mayor a cinco años. Por lo anterior asumimos que los niños han vivido en un escenario de exposición crónica a plaguicidas, más aún aquellos que acompañan a sus padres y/o algún familiar a los campos agrícolas (34.3\%) y han participado además en actividades del campo $(13.3 \%)$. El riesgo de daño a la salud se incrementa si a lo anterior se agrega que el $54.3 \%$ de los niños asistía a un centro escolar que colinda con las parcelas agrícolas, que el $42.9 \%$ de los participantes estuvieron expuestos a insecticidas empleados en el hogar o a los utilizados por el sector salud (para el control de dengue y paludismo) y/o a los agroquímicos, durante el mes previo al inicio de este estudio, y que el $39 \%$ de las madres reportó haber estado expuesta a plaguicidas durante la gestación del hijo que participó en el presente proyecto de investigación.

La edad de las madres era de 25 a 54 años, con una escolaridad promedio de $9.3 \pm 3.2$ año. El $90.5 \%$ de ellas permaneció en la zona agrícola durante sus embarazos, aproximadamente el $51 \%$ de los niños incluidos, tenían familiares que han trabajado en actividades relacionadas con la agricultura y el $54.3 \%$ reportó la existencia de campos agrícolas a menos de $15 \mathrm{~m}$ de su vivienda.

En el cuadro II se resumen los parámetros clínicos y de exposición, así como los resultados del desempeño cognitivo de los niños participantes. El IMC osciló de 12.4 a $32.5 \mathrm{~kg} / \mathrm{m}^{2}$ con una media de $17.6 \mathrm{~kg} / \mathrm{m}^{2}$. De acuerdo con los valores de corte del IMC por edad y sexo del IOTF (Cole et al. 2000, 2007), la prevalencia de sobrepeso y obesidad fue del 21 y $6.7 \%$, respectivamente. Utilizando los estándares de referencia de la OMS, los niños con puntajes $z$ del IMC para la edad mayores a +2 se clasificaron como obesos y la prevalencia fue del $6.7 \%$, en tanto que el porcentaje de niños con puntajes $z$ menores a -2 fue del $17.1 \%$, lo que significa que estos últimos tienen retraso en el crecimiento y/o bajo peso (de Onis et al. 2007).
CUADRO I. CARACTERÍSTICAS GENERALES DE LA POBLACIÓN DE ESTUDIO

\begin{tabular}{|c|c|c|}
\hline \multicolumn{3}{|l|}{ Característica } \\
\hline Edad (años) & & $9.7 \pm 1.9(5.8-14)^{\mathrm{a}}$ \\
\hline Sexo & $\begin{array}{l}\text { Femenino } \\
\text { Masculino }\end{array}$ & $\begin{array}{l}52(49.5)^{\mathrm{b}} \\
53(50.5)^{\mathrm{b}}\end{array}$ \\
\hline $\begin{array}{l}\text { Escolaridad (años) } \\
\text { Nacieron en el sitio de estudio } \\
\text { Ubicación de la escuela a la } \\
\text { que asiste el niño }\end{array}$ & $\begin{array}{l}\text { Escuela A } \\
\text { Escuela B }\end{array}$ & $\begin{array}{c}3.4 \pm 1.6(0-9)^{\mathrm{a}} \\
94(89.5)^{\mathrm{b}} \\
48(45.7)^{\mathrm{b}} \\
57(54.3)^{\mathrm{b}}\end{array}$ \\
\hline $\begin{array}{l}\text { El niño acompaña al padre } \\
\text { y/o a familiares a los campos } \\
\text { agrícolas }\end{array}$ & & $36(34.3)^{b}$ \\
\hline $\begin{array}{l}\text { El niño ayuda en actividades } \\
\text { agrícolas }\end{array}$ & & $14(13.3)^{b}$ \\
\hline $\begin{array}{l}\text { El niño ha estado expuesto a } \\
\text { insecticidas de uso doméstico, } \\
\text { empleados por el sector salud } \\
\text { y/o a agroquímicos }\end{array}$ & & $45(42.9)^{\mathrm{b}}$ \\
\hline Edad de la madre (años) & & $37 \pm 5.5(25-54)^{\mathrm{a}}$ \\
\hline Escolaridad de la madre (años) & & $9.3 \pm 3.2(1-20)^{\mathrm{a}}$ \\
\hline $\begin{array}{l}\text { La madre permaneció en el } \\
\text { sitio de estudio durante } \\
\text { el embarazo }\end{array}$ & & $41(39.0)^{b}$ \\
\hline $\begin{array}{l}\text { La madre estuvo expues- } \\
\text { ta a plaguicidas durante el } \\
\text { embarazo }\end{array}$ & & $41(39.0)^{\mathrm{b}}$ \\
\hline $\begin{array}{l}\text { El padre o un familiar es } \\
\text { agricultor }\end{array}$ & & $54(51.4)^{b}$ \\
\hline $\begin{array}{l}\text { Proximidad de campos } \\
\text { agrícolas a la vivienda }\end{array}$ & $\begin{array}{r}\leq 15 \mathrm{~m} \\
16 \mathrm{a} 60 \mathrm{~m} \\
61 \mathrm{a} 250 \mathrm{~m} \\
>251 \mathrm{~m}\end{array}$ & $\begin{array}{c}57(54.3) \\
24(22.9) \\
9(8.6) \\
15(14.3)\end{array}$ \\
\hline Ingreso familiar mensual (\$) & $\begin{array}{r}<1000 \\
1000 \text { a } 2000 \\
>2000 \mathrm{a} \\
4000 \\
>4000\end{array}$ & $\begin{array}{c}8(7.6) \\
26(24.8) \\
30(28.6) \\
37(35.2)\end{array}$ \\
\hline
\end{tabular}

${ }^{a}$ Expresada como media \pm desviación estándar (mínimo-máximo); ${ }^{b} \mathrm{n}(\%)$; escuela A: ubicada en el centro de la localidad, alejada de las parcelas; escuela B: localizada en la periferia, cerca de los campos agrícolas

El embarazo de la madre se refiere al del hijo evaluado en este estudio: $\mathrm{n}=105$

Los niños presentaron valores normales de hemoglobina, salvo una niña cuya concentración fue menor al valor de referencia $(<12 \mathrm{~g} / \mathrm{dL})$. Por otro 
lado, el valor promedio de creatinina en orina fue de $1.3 \pm 0.41 \mathrm{~g} / \mathrm{L}$ y la concentración media de plomo en sangre fue de $4.8 \pm 2.1 \mu \mathrm{g} / \mathrm{dL}(1.2$ a $10.3 \mu \mathrm{g} / \mathrm{dL})$.

Respecto a la evaluación de la exposición a plaguicidas OP, los porcentajes de detección de los metabolitos DMP, DMTP, DMDTP, DEP, DETP y DEDTP en las muestras de orina de los niños participantes $(\mathrm{n}=105)$ fueron: $62.8,99,90.4,43.8,74.3 \mathrm{y}$ $20.9 \%$, respectivamente. La media expresada como nmol/L y el mínimo y máximo de los metabolitos urinarios DMP, DEP y DAP fueron $183(<$ LD-1222), 65 (< LD-347) y 248 ( $<$ LD-1292), respectivamente (Cuadro II).

En relación con los resultados de las pruebas cognitivas que se presentan en el cuadro II, las puntuaciones promedio de los ICV, IRP, IMT e IVP fueron 89.6, 94.5, 88.2 y 94.9, respectivamente. Éstas muestran que los niños tienen mejores habilidades perceptuales que verbales y fallas en funciones ejecutivas de memoria de trabajo, concentración y velocidad de procesamiento que pueden afectar su desempeño escolar. La media del CI de los niños fue de $89.6 \pm 11.2$ y el $53.3 \%$ tuvo puntuaciones de CI menores a 90 puntos; de éstos, el $36.2 \%$ presentó valores de 80 a 89 puntos (CI promedio bajo), el $15.2 \%$ de 70 a 79 (CI límite) y el $1.9 \% \leq 69$ (CI muy bajo).
Por su parte, el $45.7 \%$ obtuvo un puntaje de 90 a 110 (CI promedio) y sólo un niño presentó un CI promedio alto (110 a 119 puntos). La distribución esperada del CI de acuerdo con los datos de referencia de la prueba es la siguiente: $25 \%$, valores menores a 90 puntos; $50 \%$, valores de 90 a 110 puntos, y $25 \%$, valores mayores a 110 (Wechsler 2003).

Las concentraciones urinarias de los metabolitos DMP, DEP y DAP expresadas en nmol/L mostraron correlaciones negativas significativas $(r=-0.204$ a $-0.345, p<0.05$, Spearman) con los ICV, IRP, IMT e IVP y con el CI (datos no mostrados en cuadros.

Otras variables que estuvieron correlacionadas con los índices cognitivos fueron la edad del niño $(r=$ $0.302, p<0.05$, Pearson) y el tiempo de residencia en la localidad ( $r=-0.238, p<0.05$, Pearson) con el IVP. Por su parte, la escolaridad de la madre se correlacionó con el IRP $(r=0.201, p<0.05$, Pearson). Aunado a lo anterior, se observó una asociación positiva entre la función intelectual de la madre con el IRP y el IVP $(r=0.264$ y 0.293 , respectivamente; $p<0.05$, Pearson).

Los niños que suelen acompañan a sus familiares a los campos agrícolas presentaron puntuaciones menores en el IVP que aquellos que no van a los campos de cultivo (91.1 y 96.9, respectivamente;

CUADRO II. INFORMACIÓN GENERAL, PARÁMETROS CLÍNICOS Y DE EXPOSICIÓN Y RESULTADOS COGNITIVOS DE LOS PARTICIPANTES

\begin{tabular}{lcccccc}
\hline & & \multicolumn{5}{c}{ Percentiles } \\
\cline { 3 - 6 } Parámetro & Media (DE) & $p 5$ & $p 25$ & $p 50$ & $p 75$ & $p 95$ \\
\hline IMC $\left(\mathrm{kg} / \mathrm{m}^{2}\right.$ ) & $17.6(3.7)$ & 13.3 & 14.5 & 16.6 & 19.5 & 25.0 \\
IMC para la edad & $0.16(1.46)$ & -2.19 & -0.85 & 0.11 & 1.37 & 2.54 \\
(puntaje $z)$ & & & & & & \\
Hemoglobina (g/dL) & $13.9(0.70)$ & 12.7 & 13.5 & 13.9 & 14.3 & 15.2 \\
Creatinina (g/L) & $1.3(0.41)$ & 0.72 & 0.93 & 1.2 & 1.5 & 2 \\
Plomo en sangre $(\mu \mathrm{g} / \mathrm{dL})$ & $4.8(2.1)$ & 2 & 3 & 4.6 & 6 & 9.6 \\
DMP (nmol/L) & $183(138)$ & 60 & 120 & 161 & 194 & 375 \\
$\quad($ nmol/g creat) & $158(106)$ & 38 & 95 & 133 & 193 & 373 \\
DEP (nmol/L) & $65(54)$ & $<\mathrm{LD}$ & 36 & 56 & 87 & 165 \\
$\quad(\mathrm{nmol} / \mathrm{g}$ creat) & $57(49)$ & $<\mathrm{LD}$ & 31 & 48 & 73 & 143 \\
DAP (nmol/L) & $248(157)$ & 60 & 155 & 231 & 301 & 493 \\
$\quad(\mathrm{nmol} / \mathrm{g}$ creat) & $216(130)$ & 38 & 132 & 198 & 255 & 472 \\
Función intelectual madre & $30.7(4.8)$ & 20.1 & 28.5 & 32 & 34 & 36 \\
ICV & $89.6(12.1)$ & 69.6 & 82 & 91 & 98.5 & 106 \\
IRP & $94.5(11.4)$ & 77 & 86 & 96 & 103 & 112 \\
IMT & $88.2(10.7)$ & 65.9 & 83 & 88 & 97 & 104 \\
IVP & $94.9(12.9)$ & 75 & 85 & 94 & 103 & 120 \\
Escala CI total & $89.6(11.2)$ & 70 & 81.5 & 89 & 99 & 108.7 \\
\hline
\end{tabular}

DMP: suma molar de los metabolitos DMP, DMTP y DMDTP; DEP: suma molar de DEP, DETP y DEDTP; DAP: suma molar de DMP y DEP; CI: coeficiente intelectual

${ }^{a}$ Evaluada mediante la prueba de matrices progresivas de Raven, $\mathrm{n}=105$ 
$p=0.025$, prueba $t$ ). Otro hallazgo importante es que los niños cuyas madres permanecieron en el sitio de estudio durante su gestación, también presentaron puntuaciones menores (93.9) del IVP, en comparación con aquellos cuyas madres vivieron fuera de la localidad agrícola durante el embarazo (104.4; $p=$ 0.037 , prueba $t$ ).

Por otro lado, las puntuaciones del ICV, del IMT y del CI fueron menores en los participantes cuya escuela colinda con los campos agrícolas, con relación a aquellos que asistían a una escuela ubicada en el centro de la localidad y por ende alejada de las parcelas agrícolas ( 86.9 vs. $92.7, p=0.014 ; 86$ vs. $91, p$ $=0.025$; y 87.5 vs. $92.2, p=0.030$, respectivamente) (datos no mostrados en cuadros).

No se observaron diferencias estadísticamente significativas entre los diferentes índices cognitivos y el CI con las variables sexo, ocupación del padre, exposición a insecticidas de uso doméstico o empleados por el sector salud y/o a agroquímicos, y la proximidad de los campos agrícolas a las viviendas.

En la figura 2 se presentan las puntuaciones de los índices cognitivos y del CI, de acuerdo con las concentraciones de los metabolitos DAP categorizadas por quintiles. En los índices de CV, RP y MT, así como en el CI, los niños con concentraciones de DAP en el primer quintil $(\leq 150.9 \mathrm{nmol} / \mathrm{L})$ tuvieron puntuaciones estadísticamente superiores a los niños de los quintiles 2 a $5(151 \mathrm{a} \leq 200.8,200.9$ a $\leq 252.7$, $252.8 \mathrm{a} \leq 312.1 \mathrm{y}>312.1 \mathrm{nmol} / \mathrm{L}$, respectivamente; ANOVA, $p<0.05$; Bonferroni, $p<0.05$ ). No se observaron diferencias estadísticamente significativas entre quintiles y las puntuaciones del índice de VP. Por otro lado, las puntuaciones de las subescalas y del CI, de acuerdo con las concentraciones urinarias de los DAP ajustadas por creatinina y categorizadas en quintiles, siguieron una tendencia similar a los valores de DAP sin ajustar, excepto en la subescala memoria de trabajo, donde no se observaron diferencias significativas entre quintiles y dicho índice (datos no mostrados). Los resultados de las pruebas cognitivas respecto de las concentraciones de metabolitos DMP y DEP ajustadas y sin ajustar por creatinina y categorizadas en quintiles, siguieron tendencias similares a las observadas en las concentraciones de los DAP (datos no mostrados en los cuadros).

En el cuadro III se muestran los MRLM de la asociación entre concentraciones (categorizadas por quintiles) de los metabolitos urinarios DMP, DEP y DAP y las diferentes puntuaciones cognitivas (subescalas y CI). Los MRLM fueron ajustados por los puntajes $z$ del IMC para la edad, el nivel de hemoglobina, el nivel de creatinina urinaria, la concentración de plomo en sangre, la función intelectual de la madre y el ingreso económico mensual familiar.

Las concentraciones urinarias de los metabolitos DAP se asociaron negativamente con las puntuaciones del ICV $(\beta=-2.6$; $95 \%$ IC: $-4.2 \mathrm{a}-1.1)$, IRP $(\beta=-2.5 ; 95 \%$ IC: -4.0 a -1.0$)$ e IMT $(\beta=-2.7$; 95\% IC: $-4.1 \mathrm{a}-1.2)$, así como con el CI $(\beta=-2.9$; 95\% IC: $-4.3 \mathrm{a}-1.5)$. La asociación inversa entre el IVP y los metabolitos DMP $(\beta=-1.9 ; 95 \%$ IC: -3.8 a -0.04$)$ y DAP $(\beta=-1.9 ; 95 \%$ IC: -3.8 a -0.06$)$ fue marginalmente significativa, mientras que no se observó una asociación significativa entre el IVP y los metabolitos DEP.

\section{DISCUSIÓN Y CONCLUSIONES}

Los resultados del presente estudio sugieren que la exposición a plaguicidas OP, estimada por las concentraciones de los metabolitos urinarios DAP, está asociada con una disminución en el desempeño cognitivo de los niños evaluados. Los niveles en orina de los DAP estuvieron inversamente asociados con las puntuaciones de los índices cognitivos y del CI, excepto con las del IVP. Los niños en el quintil Q5 de las concentraciones urinarias de la sumatoria de DAP en comparación con los ubicados en el quintil Q1 (> $312.1 \mathrm{nmol} / \mathrm{L}$ vs. $\leq 113.4 \mathrm{nmol} / \mathrm{L}$ ) presentaron una disminución de 13, 11 y 3 puntos en el ICV, el IRP y la memoria de trabajo, respectivamente.

Una limitante del presente estudio fue el empleo de los metabolitos DAP como biomarcadores de exposición a plaguicidas OP, debido a que la identificación y cuantificación de éstos no reflejan la identidad del compuesto original al que estuvieron expuestos los niños; además, pueden deberse a la degradación ambiental, incorporándose en diferentes matrices como alimentos, agua y suelo (Lu et al. 2005, Zhang et al. 2008), y estas serían las rutas de exposición. No obstante, alrededor del $80 \%$ de los plaguicidas OP se metabolizan generando estos metabolitos, por lo que conocer las concentraciones en orina de estos compuestos puede ser un marcador rentable e integral de la exposición a estos agroquímicos (Wessels et al. 2003, Barr et al., 2004, Eskenazi et al. 2004).

De acuerdo con lo indicado por pobladores de la región de estudio, los OP metilados (malatión, dimetoato, azinfos metílico y paratión metílico) son usados más frecuentemente que los OP etilados (clorpirifos, diazinón y etión). Lo anterior es consistente con los resultados obtenidos, ya que el $99 \%$ de los niños participantes presentaron concentraciones urinarias de metabolitos DMP por arriba de los 

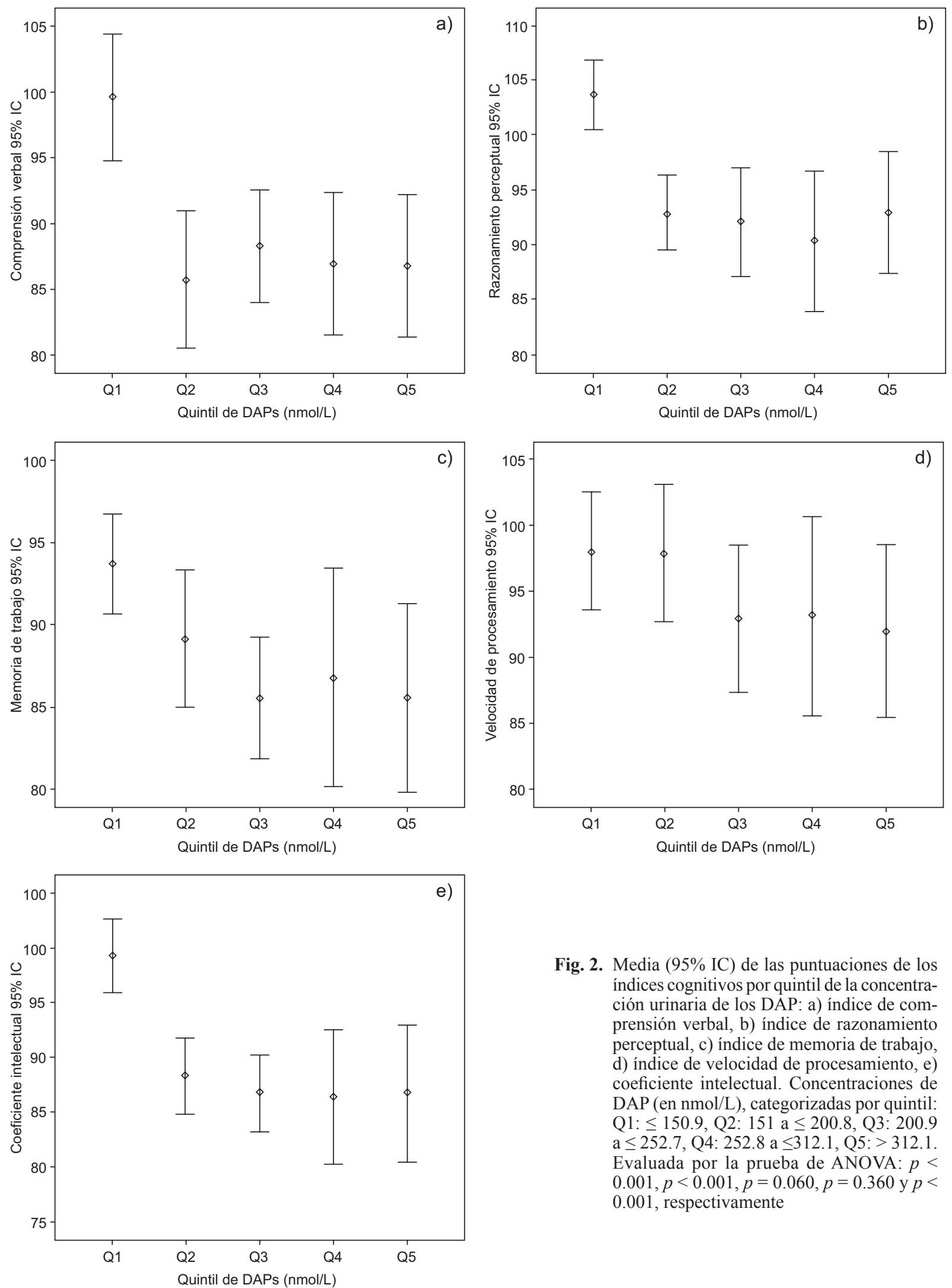

Fig. 2. Media (95\% IC) de las puntuaciones de los índices cognitivos por quintil de la concentración urinaria de los DAP: a) índice de comprensión verbal, b) índice de razonamiento perceptual, c) índice de memoria de trabajo, d) índice de velocidad de procesamiento, e) coeficiente intelectual. Concentraciones de DAP (en nmol/L), categorizadas por quintil: Q1: $\leq 150.9$, Q2: 151 a $\leq 200.8$, Q3: 200.9 $\mathrm{a} \leq 252.7, \mathrm{Q} 4: 252.8$ a $\leq 312.1, \mathrm{Q} 5:>312.1$. Evaluada por la prueba de ANOVA: $p<$ $0.001, p<0.001, p=0.060, p=0.360$ y $p<$ 0.001 , respectivamente 
CUADRO III. INFLUENCIA DE LAS CONCENTRACIONES DE LOS METABOLITOS DMP, DEP Y DAP SOBRE LAS PUNTUACIONES COGNITIVAS EN NIÑOS MEXICANOS DE UNA LOCALIDAD AGRÍCOLA*

\begin{tabular}{lccc}
\hline Índice cognitivo & $\mathrm{R}^{2}$ & $\begin{array}{c}\beta \text {-coeficiente } \\
(95 \% \mathrm{IC})\end{array}$ & $\begin{array}{c}\text { Valor } \\
\text { de } p\end{array}$ \\
\hline Comprensión Verbal & & & \\
DMP $^{\mathrm{a}}$ & 0.15 & $-2.7(-4.3 \mathrm{a}-1.0)$ & 0.002 \\
DEP $^{\mathrm{a}}$ & 0.11 & $-2.1(-3.7 \mathrm{a}-0.44)$ & 0.013 \\
DAP $^{\mathrm{a}}$ & 0.18 & $-2.6(-4.2 \mathrm{a}-1.1)$ & 0.001 \\
Razonamiento perceptual & & & \\
DMP $^{\mathrm{a}}$ & 0.22 & $-2.5(-4.0 \mathrm{a}-1.0)$ & 0.001 \\
DEP $^{\mathrm{a}}$ & 0.18 & $-1.8(-3.3 \mathrm{a}-0.28)$ & 0.021 \\
DAP $^{\mathrm{a}}$ & 0.22 & $-2.5(-4.0 \mathrm{a}-1.0)$ & 0.001 \\
Memoria de trabajo & & & \\
DMP $^{\mathrm{a}}$ & 0.20 & $-2.3(-3.8 \mathrm{a}-0.80)$ & 0.003 \\
DEP $^{\mathrm{a}}$ & 0.27 & $-3.0(-4.4 \mathrm{a}-1.6)$ & 0.000 \\
DAP $^{\mathrm{a}}$ & 0.23 & $-2.7(-4.1 \mathrm{a}-1.2)$ & 0.001 \\
Velocidad de procesamiento & & \\
DMP $^{\mathrm{a}}$ & 0.07 & $-1.9(-3.8 \mathrm{a} 0.04)$ & 0.077 \\
DEP $^{\mathrm{a}}$ & 0.07 & $-1.4(-3.3 \mathrm{a} 0.43)$ & 0.254 \\
DAP $^{\mathrm{a}}$ & 0.07 & $-1.9(-3.8 \mathrm{a}-0.06)$ & 0.075 \\
Escala CI & & & \\
DMP $^{\mathrm{a}}$ & 0.27 & $-2.9(-4.3 \mathrm{a}-1.4)$ & 0.001 \\
DEP $^{\mathrm{a}}$ & 0.22 & $-2.6(-4.1 \mathrm{a}-1.2)$ & 0.001 \\
DAP $^{\mathrm{a}}$ & 0.25 & $-2.9(-4.3 \mathrm{a}-1.5)$ & 0.001 \\
\hline
\end{tabular}

*Modelos de regresión lineal múltiples $(p<0.05)$ ajustados por función intelectual de la madre, ingreso familiar mensual, concentración de plomo en sangre, puntaje $z$ del IMC por edad, nivel de hemoglobina y nivel de creatinina

${ }^{\mathrm{a} C o n c e n t r a c i o n e s}$ en $\mathrm{nmol} / \mathrm{L}$ categorizadas por quintiles. DMP: Q1 ( $\leq 113.4)$, Q2 (113.5 a $\leq 141.2)$, Q3 (141.3 a $\leq 176.5)$, Q4 (176.6 a $\leq 207.5)$, Q5 (> 207.5); DEP: Q1 $(\leq 29.8)$, Q2 (29.9 $\mathrm{a} \leq 47)$, Q3 (47.1 $\mathrm{a} \leq 68.6), \mathrm{Q} 4$ (68.7 a $\leq$ 92.6), Q5 (>92.6); DAP: Q1 ( $\leq 150.9)$, Q2 $(151 \mathrm{a} \leq 200.8)$, Q3 $(200.9 \mathrm{a} \leq 252.7)$, Q4 (252.8 a $\leq 312.1)$, Q5 (> 312.1). DMP: suma molar de los metabolitos DMP, DMTP y DMDTP; DEP: suma molar de DEP, DETP y DEDTP; DAP: suma molar de DMP y DEP. CI: coeficiente intelectual, $\mathrm{n}=105$

límites de detección y en concentraciones promedio significativamente más altas que los metabolitos DEP (183 nmol/L vs. $65 \mathrm{nmol} / \mathrm{L})$. Dentro del grupo de los metabolitos metilados, el DMTP fue el más frecuente y presentó la concentración promedio más alta $(20.1 \mu \mathrm{g} / \mathrm{mL})$. Respecto a los etilados, el DEP fue consistentemente detectado en el $86.4 \%$ de los niños evaluados con una media de $6.9 \mu \mathrm{g} / \mathrm{mL}$.

Las concentraciones urinarias de los metabolitos DAP en los sujetos de estudio fueron superiores que las reportadas en poblaciones infantiles por otros investigadores. La media de DAP (248 nmol/L) fue dos veces mayor que la publicada por Barr et al. (2004), quienes evaluaron a niños de una población abierta de EUA; Oulhote y Bouchard (2013) detectaron concentraciones aproximadamente 2.3 veces menores en niños canadienses de 6 a 11 años de edad.

Respecto a estudios realizados en poblaciones infantiles de comunidades agrícolas, destacan los trabajos de Curl et al. (2002), Koch et al. (2002) y Bradman et al. (2013); en todos ellos, el valor de la media de DAP fue 1.5 a 1.7 veces menor que la detectada en este estudio. Solo Koch et al. (2002) mencionan que las muestras de orina fueron recolectadas durante el periodo de aplicación de los plaguicidas.

Lambert et al. (2005), quienes estudiaron a niños de 2 a 6 años de edad de tres diferentes comunidades agrícolas de Oregon, también mencionan que los metabolitos metilados fueron los encontrados con mayor frecuencia, aunque con concentraciones de 1.4 a 2.7 veces menores que las cuantificadas en este estudio (media $183 \mathrm{nmol} / \mathrm{L}$ vs. 68 a $130 \mathrm{nmol} / \mathrm{L}$ ).

La variabilidad en las concentraciones de los metabolitos DAP reportadas entre los estudios previamente citados y el presente podría deberse a diferencias en los patrones de uso y aplicación de los plaguicidas (tipos, cantidades y periodos de aplicación), a las condiciones climáticas que influyen en la degradación y permanencia de residuos de los agroquímicos, a las características del sitio de estudio (área urbana o rural, actividades productivas, proximidad de campos agrícolas a las viviendas) y a las características de la población valorada (edad, estado nutricio, tiempo de residencia en la localidad, exposición prenatal, ocupacional, no ocupacional o residencial, así como a la variabilidad intraindividuos) (Au et al. 2001, Fenske et al. 2005, Vida y Moretto 2007, Muñoz-Quezada et al., 2012).

El interés de evaluar la neurotoxicidad producida por la exposición crónica a plaguicidas OP va en aumento, debido a las observaciones epidemiológicas de que los niños expuestos prenatalmente o durante la vida postnatal temprana, presentan diversos déficits neurológicos (Eskenazi et al. 2004, 2007, 2010, Young et al. 2005, Rauh et al. 2006, Handal et al. 2007a, 2007b, 2008, Marks et al. 2010, Bouchard et al. 2011, Quirós et al. 2011, Yolton et al. 2013). Sin embargo, pocos estudios han valorado si el neurodesarrollo de estos niños en etapas posteriores (infancia y adolescencia) puede verse afectado por dicha exposición (Guillette et al. 1998, Ruckart et al. 2004, Rohlman et al. 2005, Grandjean et al. 2006). De ahí la relevancia del presente estudio, en el cual fueron evaluados 105 niños de 5 a 14 años de edad 
de una comunidad agrícola, que han estado expuestos a plaguicidas desde su gestación o su nacimiento. Entre los resultados más relevantes está el que los participantes clasificados en el quintil con la mayor concentración de DAP presentaron un déficit de 12 puntos en el CI, en comparación con los niños del quintil con la menor concentración $(>312.1 \mathrm{nmol} / \mathrm{L}$ vs. $\leq 150.9 \mathrm{nmol} / \mathrm{L}$ ).

De las subescalas evaluadas, el IMT presentó las puntuaciones más bajas (media: 88.2) y su asociación inversa con los niveles urinarios de los DAP fue altamente significativa (DMP: $p=0.003$; DEP: $p=0.000$; DAP: $p=0.001$ ). La memoria de trabajo es la capacidad para mantener información de forma activa, mediante procesos neurobiológicos de almacenamiento y de recobro de la información básica en el aprendizaje y en el pensamiento; sus alteraciones pueden influir en diversos procesos formales de aprendizaje académico, tales como dificultad para enfocar la atención, dificultad para el reconocimiento de patrones de prioridad, incapacidad para reconocer las jerarquías y dificultad para el reconocimiento y selección de los objetivos más adecuados para la resolución de un problema (Etchepareborda y Abad-Mas 2005). Por ello los resultados aquí obtenidos sugieren que los niños de localidades agrícolas expuestos a OP podrían estar en mayor riesgo de presentar alteraciones cognitivas, tal como lo mencionan Guillete et al. (1998), quienes reportaron que niños residentes en el Valle del Yaqui, Sonora, México, y por lo tanto expuestos a mezclas de plaguicidas, presentaron alteraciones tanto en su coordinación gruesa como fina y en la memoria a corto plazo. Ruckart et al. (2004) también detectaron que aquellos niños que estaban expuestos a metil paratión presentaron mayor dificultad para desempeñar tareas que implicaban memoria a corto plazo.

Por otro lado, en un estudio realizado con niños de edad preescolar habitantes de comunidades agrícolas y no agrícolas, Rohlman et al. (2005) observaron que aquellos infantes con exposición crónica a agroquímicos presentaron menor rendimiento en los dominios de velocidad de respuesta y latencia en comparación con los niños no expuestos. Estos resultados concuerdan con lo reportado por Grandjean et al. (2006), quienes evaluaron a hijos de floricultores y encontraron que los niños expuestos a plaguicidas OP tuvieron los puntajes más bajos de la prueba de copiado de Stanford-Binet, así como un tiempo de reacción simple mayor.

El estudio de Muñoz et al. (2011) reportó una correlación inversa ( $r=0.44 ; p=0.014$ unilateral $)$ entre el IVP y las concentraciones del metabolito DMTP en niños chilenos de 6 a 11 años de edad de escuelas rurales, a pesar de que los niveles urinarios de este metabolito fueron inferiores $(2.5$ a $51.4 \mu \mathrm{g} / \mathrm{L})$ a los detectados en los infantes de este trabajo $(<\mathrm{LD}$ a $121.4 \mu \mathrm{g} / \mathrm{L}$ ). Los resultados muestran una asociación inversa (-1.9) con significancia limitada $(p=0.075$, prueba bilateral) entre los niveles de DMTP y el IVP.

Lizardi et al. (2007), quienes evaluaron a niños de 7 años de edad de una localidad agrícola ubicada al sur de Arizona, reportaron dos comportamientos en función de las concentraciones urinarias de los DAP: a concentraciones bajas (cerca del valor del LD) no observaron asociación alguna con el desempeño cognitivo, mientras que a concentraciones altas (media igual a $110 \mu \mathrm{g} / \mathrm{mL}$ ) observaron una asociación negativa. La media de los niveles urinarios de estos metabolitos en los niños del presente estudio fue de $36 \mu \mathrm{g} / \mathrm{mL}$, notoriamente menor a la detectada por Lizardi et al. (2007).

Se ha reportado que el CI es un predictor del nivel de logro académico de los escolares; no obstante, también se ha señalado que no es la única medida para el éxito (Festus 2012). Braaten y Norman (2006) comentan que el CI está influenciado por diversos factores y sugieren que una parte significativa de la variación observada es de origen genético, en tanto que Eckerman et al. (2007) mencionan que componentes como la exposición a drogas y contaminantes, el nivel socioeconómico, el entorno familiar, la educación y el CI de los padres, además de una nutrición adecuada, la intervención de los padres en el aprendizaje de sus hijos y situaciones de estrés o dificultades emocionales, entre otros, pueden influir en los resultados de las pruebas cognitivas. De ahí que una de las fortalezas del presente estudio fue la inclusión de factores confusores en la valoración del efecto neurotóxico por la exposición infantil a plaguicidas OP, tales como los niveles sanguíneos de plomo, el nivel de hemoglobina, la escolaridad y la función intelectual de la madre, el ingreso económico mensual familiar y el estado nutricional de los niños (estimado mediante los valores de puntaje $z$ del IMC para la edad).

Alrededor del $60 \%$ de los niños participantes en este estudio, tuvieron una concentración media de plomo similar a la media nacional (4.8 vs. $4.5 \mu \mathrm{g} / \mathrm{dL})$, la cual se obtuvo a partir de un biomonitoreo realizado en 10 estados de México (Díaz-Barriga 2004). En México, la gasolina sin este metal y el uso de pintura libre de plomo ha contribuido a la reducción de la exposición a este elemento; sin embargo, otras fuentes de exposición continúan siendo un riesgo para la salud de la población, tal es el caso del empleo de 
loza vidriada y el consumo de alimentos y dulces contaminados con este metal (Caravanos et al. 2014). En el presente estudio no se identificaron las fuentes potenciales ni las rutas de exposición al plomo, ni se encontró una asociación entre éste y las subescalas cognitivas y el CI. Estos hallazgos no coinciden con los resultados obtenidos en un estudio realizado en la misma comunidad, hace aproximadamente seis años por el mismo grupo de investigación (Rivero-Pérez 2012). En ese tiempo se detectaron concentraciones de plomo por arriba del valor de referencia $(5 \mu \mathrm{g} / \mathrm{dL}$ vs $6.3 \mu \mathrm{g} / \mathrm{dL}$ ) en el $60 \%$ de los niños evaluados, y se observó una asociación significativa entre los niveles de este neurotóxico y los Índices de CV y RP. Una explicación podría ser que en las inmediaciones de la escuela a la cual asistían estos niños (es importante resaltar que no es la misma a la que pertenecen los niños del presente estudio) se identificaron varias ladrilleras, en las cuales se emplean como combustibles aceite quemado, llantas y residuos de aparatos eléctricos y electrónicos.

Si bien es cierto que diversos autores han demostrado efectos en el CI y de tipo cognitivo en poblaciones infantiles expuestas a plaguicidas OP, estos estudios fueron realizados en poblaciones de otros países. Respecto de los reportes en niños mexicanos, la estimación de la exposición a agroquímicos se asumió con base en información recabada en entrevistas o por las características del sitio de estudio (zonas agrícolas vs. urbanas). Contrario a ello, en el presente trabajo se cuantificaron en orina los seis principales metabolitos DAP por cromatografía de gases-masas para evaluar la magnitud de la exposición infantil a plaguicidas OP, por lo que una fortaleza más de este estudio es haber evaluado de forma objetiva y analítica la dimensión de la exposición infantil a estos agroquímicos, para posteriormente relacionarla con su desempeño cognitivo.

Van Thriel et al. (2012), han propuesto modelos de genética de imágenes e in vitro para tratar de explicar las correlaciones neurobiológicas de la función cognitiva con la exposición a bifenilos policlorados y a plaguicidas OP, sugiriendo que estas alteraciones pueden deberse a la pérdida de la plasticidad sináptica, alteraciones en el receptor del glutamato (NMDA-D aspartato), y cambios en la morfología axonal y dendrítica, lo que alteraría tanto la conectividad neuronal como la formación de redes neuronales en superficies estructuradas.

En conclusión, no obstante que la estimación de la exposición a estos agroquímicos se realizó en el periodo de alta aplicación de los mismos, los niños del estudio han estado expuestos crónicamente a estos contaminantes desde su gestación o nacimiento, lo que explicaría la asociación negativa de la concentración urinaria de la sumatoria de metabolitos DAP con su desempeño cognitivo. Para valorar si estas alteraciones cognitivas pueden repercutir en el desempeño de estos niños, se sugiere diseñar estudios de seguimiento a corto y largo plazo.

\section{REFERENCIAS}

Androutsopoulos V.P., Hernandez A.F., Liesivuori J. y Tsatsakis A.M. (2012). A mechanistic overview of health associated effects of low levels of organochlorine and organophosphorous pesticides. Toxicology 307, 89-94. DOI: 10.1016/j.tox.2012.09.011.

Arcury T.A., Grzywacz J.G., Barr D.B., Tapia J., Chen H. y Quandt S.A. (2007). Pesticide urinary metabolite levels of children in Eastern North Carolina farmworker households. Environ. Health Perspect. 115, 1254-1260. DOI: 10.1002/ajim.20354

Au W.W., Oh H.Y., Grady J., Salama S.A. y Heo M.Y (2001). Usefulness of genetic susceptibility and biomarkers for evaluation of environmental health risk. Environ. Mol. Mutagen. 37, 215-225.

DOI: $10.1002 / \mathrm{em} .1030$

Barr D.B., Bravo R., Weerasekera G., Caltabiano L.M., Whitehead R.D., Olsson, A. O., Caudill S.P., Schober S.E., Pirkle J.L., Sampson E.J., Jackson R.J.J. y Needham L.L.G. (2004). Concentrations of dialkyl phosphate metabolites of organophosphorus pesticides in the U.S. population. Environ. Health Perspect. 112, 186-200.

Bouchard M.F., Chevrier J., Harley K.G., Kogut K., Vedar M., Calderon, N., Trujillo C., Johnson C., Bradman A., Barr D.B. y Eskenaz B. (2011). Prenatal exposure to organophosphate pesticides and IQ in 7-year-old children. Environ. Health Perspect. 119, 1189-1195. DOI: 10.1289/ehp.1003185

Braaten E.B. y Norman D. (2006). Intelligence (IQ) testing. Pediatrics Rev. 27, 403-408.

DOI: 10.1542/pir.27-11-403

Bradman A., Kogut K., Eisen E.A., Jwell N.P., QuirósAlcalá L., Castorina R, Chevrier J., Holland N.T., Barr D.B., Kavanagh-Baird G. y Eskenazi B. (2013). Variability of organophosphorous pesticide metabolite levels in spot and 24-hr urine samples colleted from young children during 1 week. Environ. Health Perspect. 121, 118-124.

Caravanos J., Dowling R., Téllez-Rojo M.M., Cantoral A., Kobrosly R., Estrada D., Orjuela M., Gualtero S., Ericson B., Rivera A y Fuller R. (2014). Niveles de plomo en sangre en méxico y su implicación para la 
carga pediátrica de la enfermedad. Ann. Global Health 80, 1-11. DOI: 10.1016/j.aogh.2014.10.005

Cole T.J., Bellizzi M.C., Flegal K.M. y Dietz W.H. (2000). Establishing a standard definition for child overweight and obesity: international survey. BMJ-Brit. Med. J. 320, 1240-1243.

DOI: $10.1136 /$ bmj.320.7244.1240

Cole T.J., Flegal K.M., Nicholls D. y Jackson A.A. (2007). Body mass index cut-offs to define thinness in children and adolescents: international survey. BMJ-Brit. Med. J. 335, 194-202. DOI: 10.1136/bmj.39238.399444.55

Costa L.G., Cole T.B., Jarvik G.P. y Furlong C.E. (2003). Functional genomics of the paraoxonase (PON1) polymorphisms: effects on pesticide sensitivity, cardiovascular disease, and drug metabolism. Annu. Rev. Med. 54, 371-392.

DOI: 10.1146/annurev.med.54.101601.152421

Costa L.G., Cole T.B., Vitalon A. y Furlong C.E. (2005). Measurement of paraoxonase (PON1) status as a potential biomarker of susceptibility to organophosphate toxicity. Clin. Chimica Acta 352, 37-47.

DOI: $10.1016 /$ j.ccen.2004.09.019

Costa L.G. (2013). Toxic effects of pesticides. En: Casarett and Doull's toxicology: The basic science of poisons, 8a ed. (Klaassen C.D., Ed.), cap. 22.

Curl C.L., Frenske R.A., Kissel J.C., Shirai J.H., Moate T.F., Griffith W., Coronado G. y Thompson B. (2002). Evaluation of take-home organophosphorus pesticide exposure among agricultural workers and their children. Environ. Health Perspect. 110, 829-833.

DOI: 10.1289/ehp.021100787

Díaz-Barriga F. (2004). Metales y contaminantes orgánicos persistentes en niños y muestras ambientales de 10 sitios contaminados. Instituto Nacional de Ecología, México.

De Onis M., Onyango A.W., Borghi E., Siyam A., Nishida C. y Siekmann J. (2007). Development of a WHO growth reference for school-aged children and adolescents. Bull. WHO 85, 660-667.

DOI: 10.2471/BLT.07.043497

Eckerman D.A., Gimenes L.S., Curi de Souza R., Lopes Galvão P.R., Sarcinelli P.N. y Chrisman J.R. (2007). Age-related effects of pesticide exposure on neurobehavioral performance of adolescent farm workers in Brazil. Neurotox. Terat. 29, 164-175.

DOI: 10.1016/j.ntt.2006.09.028

Engel S.M., Berkowitz G.S., Barr D.B., Teitelbaum S.L., Siskind J., Meisel S.J., Wetmur J.G. y Wolff M.S. (2007). Prenatal organophosphate metabolite and organochlorine levels and performance on the Brazelton Neonatal Behavioral Assessment Scale in a multiethnic pregnancy cohort. Am J. Epidemiol. 165, 1397-1404. DOI: $10.1093 /$ aje/kwm029
Engel S.M., Wetmur J., Chen J., Zhu C., Barr D.B., Canfield R.L. y Wolf M.S. (2011). Prenatal exposure to organophosphates, paraoxonase 1 , and cognitive development in childhood. Environ. Health Perspect. 119, 1182-1188. DOI: 10.1289/ehp.1003183

Eskenazi B., Harley K., Bradman A., Weltzien E., Jewell N.P., Barr D.B., Furlong C.E. y Holland N.T. (2004). Association of in utero organophosphate pesticide exposure and fetal growth and length of gestation in an agricultural population. Environ. Health Perspect. 112, 1116-1124. DOI : 10.1289/ehp.6789

Eskenazi B., Marks A.R., Bradman A., Harley K., Barr D.B., Johnson C., Morga M. y Jewell N.P. (2007). Organophosphate pesticide exposure and neurodevelopment in young Mexican-American children. Environ. Health Perspect. 115, 792-798.

DOI: $10.1289 /$ ehp. 9828

Eskenazi B., Huen K., Marks A., Harley K.G., Bradman A., Barr D.B. y Holland, N. (2010). PON1 and neurodevelopment in children from the CHAMACOS study exposed to organophosphate pesticides in utero. Environ. Health Perspect. 118, 1775-1781.

DOI: 10.1289/ehp.1002234

Etchepareborda M.C. y Abad-Mas L. (2005). Memoria de trabajo en los procesos básicos del aprendizaje. Rev. Neurol. 40 (Supl. 1, S79-S83).

Festus A.B. (2012). The relationship between emotional intelligence and academic achievement of senior secondary school students in the federal capital territory, Abuja. Journal of Education and Practice 3, 13-19. DOI: $10.17265 / 2159-5542 / 2016.10 .002$

Fenske R.A., Lu C., Curl C.L., Shirai J.H. y Kissel J.C. (2005). Biologic monitoring to characterize organophosphorus pesticide exposure among children and workers: An analysis of recent studies in Washington state. Environ. Health Perspect. 113, 1651-1657.

DOI: $10.1289 / \mathrm{ehp} .8022$

Gamlin J., Díaz, Romo P. y Hesketh T. (2006). Exposure of young children working on Mexican tobacco plantations to organophosphorous and carbamic pesticides, indicated by cholinesterase depression. Child Care Hlth. Dev. 33, 246-248.

DOI: $10.1111 / \mathrm{j} .1365-2214.2006 .00702 . x$

Grandjean P., Harari R., Barr D.B. y Debes F. (2006). Pesticide exposure and stunting as independent predictors of neurobehavioral deficits in Ecuadorian school children. Pediatrics 117, 546-556.

DOI: $10.1542 /$ peds.2005-178

Guillette E.A., Meza M.M., Aguilar M.G., Soto A.D y García I.E. (1998). An anthropological approach to the evaluation of preschool children exposed to pesticides in Mexico. Environ. Health Perspect. 106, 347-353. DOI: $10.1289 /$ ehp. 98106347 
Handal A.J., Lozoff B., Breilh J. y Harlow S.D. (2007a). Neurobehavioral development in children with potential exposure to pesticides. Epidemiology 18, 312-320. DOI: 0.1097/01.ede.0000259983.55716.bb

Handal A.J., Lozoff B., Breilh J. y Harlow S.D. (2007b). Effect of community of residence on neurobehavioral development in infants and young children in a flowergrowing region of Ecuador. Environ. Health Perspect. 115, 128-133. DOI: 10.1289/ehp.9261

Handal A.J., Harlow S.D., Breilh J. y Lozoff B. (2008). Occupational exposure to pesticides during pregnancy and neurobehavioral development of infants and toddlers. Epidemiology 19, 851-859.

DOI: 10.1097/EDE.0b013e318187cc5d

INEGI (2010). Instituto Nacional de Estadística y Geografía. Censo de Población y Vivienda 2010. Disponible en: http://www.inegi.gob.mx.

Kamel F., Engel L.S., Gladen B.C., Hoppin J.A., Alavanja M.C. y Sandler D.P. (2007). Neurologic symptoms in licensed pesticide applicators in the agricultural health study. Hum. Exp. Toxicol. 26, 243--250.

DOI: $10.1177 / 0960327107070582$

Koch D., Lu C., Fisker-Andersen J., Jolley L. y Fenske R.A. (2002). Temporal association of children's pesticide exposure and agricultural Spraying: Report of a longitudinal biological monitoring study. Environ. Health Perspect. 110, 829-833.

DOI: $10.1289 / \mathrm{ehp} .02110829$

Lambert W.E., Lasarev M., Muñiz J., Scherer J., Rothlein J., Santana J y McCaule J. (2005). Variation in organophosphate pesticide metabolites in urine of children living in agricultural communities. Environ. Health Perspect. 113, 504-508. DOI: 10.1289/ehp.6890

Lizardi P.S., O'Rourke M.K. y Morris R.J. (2007). The effects of organophosphate pesticide exposure on Hispanic children's cognitive and behavioral functioning. J. Pediatr. Psychol. 33, 91-101. DOI:10.1093/jpepsy/jsm047

Lu C., Bravo R., Caltabiano L.M., Irish R.M., Weerasekera G. y Barr D.B. (2005). The presence of dialkylphosphates in fresh fruit juices: implication for organophosphorus pesticide exposure and risk assessments. J. Toxicol. Environ. Health A 68, 209-227. DOI: $10.1080 / 15287390590890554$

Marks A.R., Harley K., Bradman A., Kogut K., Barr D.B., Johnson C., Calderon N. y Eskenazi B. (2010). Organophosphate pesticide exposure and attention in young Mexican-American children: The CHAMACOS study. Environ. Health Perspect. 118, 1768-1774). DOI: $10.1289 /$ ehp.1002056

Mink P., Goodman M., Barraj L., Imrey H., Kelsh M. y Yager J.(2004). Evaluation of uncontrolled confounding in studies of environmental exposures and neurobehavioral testing in children. Epidemiology 15(4),385-393.

DOI: $10.1097 / 01 . e d e .0000128402 .86336 .7 \mathrm{e}$

Muñoz M.T., Iglesias V.P. y Lucero B.A. (2011). Exposure to organophosphate and cognitive performance in Chilean rural schoolchildren: an exploratory study. Rev. Fac. Nac. Salud Pública 29(3), 256-263.

Muñoz-Quezada M.T., Iglesias V., Lucero B., Steenland K., Barr D.B., Levy K., Ryand P.B., Alvarado S. y Concha C.E. (2012). Predictors of exposure to organophosphate pesticides in schoolchildren in the Province of Talca, Chile. Environ. Int. 47, 28-36.

DOI: $10.1016 /$ j.envint.2012.06.002

Oulhote Y. y Bouchard M.F. (2013). Urinary metabolites of organophosphate and pyrethroid pesticides and behavioral problems in Canadian children. Environ. Health Perspect. 121, 1378-1384.

DOI: 10.1002/9780470640500.ch34

Quintanilla-Vega B., Pérez-Herrer, N. y Rojas-García E. (2010). Epidemiological studies of anticholinesterase pesticide poisoning in Mexico. En: Anticholinesterase pesticides: Metabolism, neurotoxicity, and epidemiology. (Satoh T. y Gupta R.C., Eds.). John Wiley and Sons, 471-479.

Quirós-Alcalá L., Alkon A.D., Boyce W.T., Lippert S., Davis N.V., Bradman A., Barr, D.B. y Eskenazi B. (2011). Maternal prenatal and child organophosphate pesticide exposures and children's autonomic function. Neurotoxicology 32, 646-655.

DOI: $10.1016 /$ j.neuro.2011.05.017

Ramírez-Jiménez M.R. (2015). Asociación entre la exposición a plaguicidas organofosforados y la paraoxonasa 1 (PON1) y alteraciones neurocognitivas en niños y adolescentes de una comunidad agrícola de SLP. Tesis de Doctorado. Universidad Autónoma de San Luis Potosí, México, 149 pp.

Rauh V.A., Garfinkel R., Perera F.P., Andrews H.F., Hoepner L., Barr D.B., Whitehead R., Tang D. y Whyatt R.W. (2006). Impact of prenatal chlorpyrifos exposure on neurodevelopment in the first 3 years of life among inner-city children. Pediatrics 118, 1845-1859.

DOI: $10.1542 /$ peds.2006-0338

Raven J.C. (1993). Test de matrices progresivas para la medida de la capacidad intelectual de sujetos de 12 a 65 años. Paidós, México.

Rivero-Pérez N.E. (2012). Evaluación de los efectos en salud por la exposición a plaguicidas en niños de San Luis Potosí. Tesis de Doctorado. Universidad Autónoma de San Luis Potosí, México, 191 pp.

Rohlman D.S., Arcury T.A., Quandt S.A., Lasarev M., Rothlein J., Travers R., Tamulinas A., Scherer J., Early J., Marín A., Phillips J. y McCauley L. (2005). Neurobehavioral performance in preschool children 
from agricultural and non-agricultural communities in Oregon and North Carolina. Neurotoxicology 26, 589-598. DOI: 10.1016/j.neuro.2004.12.002

Ruckart P.Z., Kakolewski K., Bove F.J. y Kaye W.E. (2004). Long-term neurobehavioral health effects of methyl parathion exposure in children in Mississippi and Ohio. Environ. Health Perspect. 112, 46-51. DOI:10.1289/ehp.6430

Sánchez-Guerra M., Pérez-Herrera N., y Quintanilla-Vega B. (2011). Organophosphorous pesticides research in Mexico: epidemiological and experimental approaches. Toxicol. Mech. Methods 21(9), 681-691. DOI: $10.3109 / 15376516.2011 .602130$

Sly P.D. y Flack F.(2008). Susceptibility of children to environmental pollutants. Ann. N.Y. Acad. Sci. 1140, 163-183. DOI: 10.1196/annals.1454.017

Subramanian K.S. (1987). Determination of lead in blood: Comparison of two GFASS methods. Atomic Spectrosc. 8, 7-14. DOI: 10.1016/S0946-672X(02)80037-2

Valcke M., Samuel O., Bouchard M., Dumas P., Belleville D. y Tremblay C. (2006). Biological monitoring of exposure to organophosphate pesticides in children living in peri-urban areas of the Province of Quebec, Canada. Int. Arch. Occup. Environ. Health 79, 568577. DOI: $10.1007 / \mathrm{s} 00420-006-0085-8$

Van Thriel Ch., Westerink R.H.S., Beste CH., Bale A.S., Lein P.J. y Leist M. (2012). Translating neurobehavioural endpoints of developmental neurotoxicity test into in vitro assays and readouts. Neurotoxicology 33, 911-924.

DOI: 10.1016/j.neuro.2011.10.002
Vida P. y Moretto A. (2007). Pesticide exposure pathways among children of agricultural workers. J. Public Health 15, 289-299. DOI: 10.1007/s10389-007-0127-z Wechsler D. (2003). WISC-IV administration and scoring manual (Wechsler intelligence children, 4a. ed.). Harcourt Assessment, San Antonio, 272 pp.

Weiss B., Amier S. y Amier R.W. (2004). Pesticides. Pediatrics 113, 1030-1038. DOI: 10.1542/peds.113.1.134

Wessels D. Barr D.B. y Mendola P. (2003). Use of biomarkers to indicate exposure of children to organophosphate pesticides: Implications for a longitudinal study of children's. Environ. Health Perspect. 111, 1939-1946. DOI: 10.1289/ehp.6179

Yolton K., Xu Y., Sucharew H., Succop P., Altaye M., Popelar A., Montesano M.A., Calafat A.M. y Khoury J.C. (2013). Impact of low-level gestational exposure to organophosphate pesticides on neurobehavior in early infancy: A prospective study. Environ. Health 12, 79. DOI: 10.1186/1476-069X-12-79

Young J.G., Eskenazi B., Gladstone E.A., Bradman A., Pedersen L., Johnson C., Barr D.B. Furlong C.E. y Holland N.T. (2005). Association between in utero organophosphate pesticide exposure and abnormal reflexes in neonates. Neurotoxicology 26, 199-209. DOI: $10.1016 /$ j.neuro.2004.10.004

Zhang, X., Driver J.H., Li Y., Ross J.H. y Krieger R. I. (2008). Dialkylphosphates (DAPs) in fruits and vegetables may confound biomonitoring in organophosphorus insecticide exposure and risk assessment. J. Agric. Food Chem. 56, 10638-10645.

DOI: $10.1021 / \mathrm{jf} 8018084$ 